\title{
Cochlear Implants for Sensorineural Hearing Loss
}

\author{
${ }^{1}$ Goutam Goyal, ${ }^{2}$ Dr. K.K. Dhawan \\ ${ }^{I}$ M.E.,Biomedical Engineering Research Group, Singhania University Rajastha. \\ , ${ }^{2}$ Ph.D. Director, Shekhawati Group Of College Shekhawati, Rajastha.,
}

\begin{abstract}
This article briefly discuses the types and effects of hearing loss and then focuses on the topic of cochlear implants. The history of such devices is reviewed, as are their components and functions. Selection criteria for adult and pediatric implant candidates are outlined, and the procedures, risks, follow up, and likely outcomes of surgery are examined. The controversial issue of providing implants to children also addressed.
\end{abstract}

\section{Sensitivity and Acuity}

\section{Hearing Loss}

Hearing loss comes in many forms and degrees of severity. Auditory disorders can result in decreased sensitivity, decreased acuity (clarity), or both. With decreased sensitivity, sounds are simply too soft. If sounds are made louder, the listener is able to understand speech with little difficulty. Generally, the degree of hearing loss in cases of decreased sensitivity is not extreme.

Conductive hearing loss, which involves disorders of the outer and/or middle ear, most often results in reduced sensitivity, with acuity remaining good. Hearing aids work quite well for patients with conductive hearing losses. Often, conductive hearing losses are medically or surgically correctable.

Auditory disorders also can occur in either sensory or neural portions of the auditory system. Disorders in these areas typically result in loss of acuity as well as loss of sensitivity. Sensory receptors of the cochlea may be damaged or destroyed. when damage to the nerve or loss of sensory cells becomes too great, portions of the signal may be lost completely. Consequently, no matter how loud the sound is made through a hearing aid, the listener is unable to understand the words. This situation is analogous to a radio station that is not tuned properly. No matter how loud the volume, clarity is not improved; indeed, increasing the volume may decrease some patients ability to understand.

Losses in both sensitivity and acuity may range from mild to total. Some people with less severe sensorineural hearing loss are able to derive adequate benefit from hearing aids. For both adults and children whose sensorineural damage is too great, however, coping with deafness and learning to communicate visually were for many years the only options.

\section{Effects of Hearing Loss}

It is well documented that childhood deafness can have a severe impact on speech and language development, which can result its emotional, social, educational, and vocational disruption as the child matures. ${ }^{2}$ Language and in our society, oral language is the primary means through which socialization and learning occur. Development of speech and language occurs rapidly in the first few years of life, primarily through normal family interaction, If the communication interaction between child and family is disrupted during these early critical years, serious delays are likely to occur. If the deprivation goes on for too long, the child may never make up the lost learning, even with extensive rehabilitation. Supporting this claim, the average reading level of deaf 18 year old persons is just below the third grade level. ${ }^{3}$ Adults who have been deaf since childhood tend to be undereducated and earn less money, compared with their hearing peers. ${ }^{4}$ Severe to profound hearing loss has the potential to adversely affect many aspects of development, including social, cognitive, and academic abilities, primarily because of language delay. ${ }^{5}$ In the long term, deficits in these areas can limit vocational and economic potential.

Loss of hearing in the adult years is only slightly less devastating. Withdrawal from family and friends, loss of career, and increased feelings of insecurity are all potential effects of hearing loss in adults. Ramdell ${ }^{6}$ distinguishes 3 levels of hearing. The first level is basic awareness. Whether or not we realize it, hearing persons are constantly in touch with our environment auditor ally. Without this constant contact with the environment, deafened persons often report either feeling as though they are walking around "dead" or feeling alone in the world. The second level of hearing is the warning level. In this level, sound acts as a sign or signal of events to which we make constant adjustments in our daily living. ${ }^{6}$ allowing us, for example, to recognize that we should leave the building if the fire alarm is sounding, pull over to the right or the road if we hear a siren, or answer the telephone if it is ringing. Loss at this level decreases a person's feeling of safety and security. Finally, the third and highest level of hearing is that used socially for comprehension of language. In this symbolic level, spoken words take on meaning. To hear and understand "I love you" is an ability shared by no other species. Although 
most animal species communicate at some level, only humans have the ability to use words to describe their thoughts and feelings. This level of hearing separates humans from other species. To lose this level of hearing is to feel isolated and, perhaps, even less human. The experience of hearing is a combination of all of these levels, which occur, essentially, simultaneously.

\section{Cochlear Implants}

Cochlear implants are essentially neural stimulators, which, when implanted into the cochlea of the inner ear, bypass the function of the sensory receptors. Minute electrical curents directly stimulate ganglion cells of the auditory nerve and are transmitted by the auditory nerve to the auditory cortex, where they are interpreted as sound. There are approximately 20,000 sensory receptors in the normal cochlea. Cochlear implants replace the function of damaged receptors with anywhere from 8 to 24 stimulating contacts.

\section{History and Development}

The concept of using electrical current to stimulate the auditory system dates back to the 1780 s, when Volta attempted to stimulate his own auditory system by placing battery contacts into his ear canals. This idea was resurrected in the 1950s by the French surgeons Djourno and Eyries, who were able to elicit a hearing sensation in a deaf person using an electrode placed into the cochlea after surgery. In the early 1970s, Dr. William House of the House Ear Institute in Los Angeles developed a practical device that could be worn by patients outside the laboratory. This device used a single ball electrode placed approximately $6 \mathrm{~mm}$ into the cochlea. Activation of the electrode stimulated all remaining neural elements within its current range in an analog representation of the incoming sound. the result was a good reproduction of temporal characteristics of speech but provided very little information regarding spectral (pitch) content. In other words, the patient essentially heard a buzz with the same intonation pattern as the speech stimulus. However, even this limited information proved a valuable asset to speech reading in totally deaf individuals. Receiving at least some auditory awareness was reported by patients to increase feelings of security in the environment and lessen feelings of isolation and depression. There was, however, little understanding of speech without concurrent visual information. ${ }^{7}$

However, it was during the 1990s that cochlear implant technology saw its greatest leaps forward. Improved internal electrode design was aimed at enabling surgeons to place the device consistently closer to the modulus, where the remaining stimulate elements are located. External device continued to become smaller and, at the same time, more flexible.

\section{Components and Function}

All cochlear implant systems have both internal and external components. The external components include a wearable microcomputer (speech processor), a microphone, and a radiofrequency transmitting coil. The microphone, usually worn at or near the ear, picks up incoming sounds. From the microphone, sound travels along a cable to the speech processor, where acoustic information representing key aspects of speech is analyzed. These aspects are coded as frequency (i.e., electrode selection) and intensity i.e., current amplitude). The electrical code is sent across the skin by a radio wave, directing the function of the internal component.

The internal component, called the receiver stimulator or implantable cochlear stimulator, comprises a computer chip and housing, radiofrequency-receiving coil, and stimulating electrodes placed into a solstice carrier. This is the portion that is implanted by the surgeon and is intended to remain in place for a lifetime.

The signal sent by the speech processor via the transmitting coil is picked up by the radiofrequency-receiving coil and sent to the computer chip. The appropriate electrode along the array is selected and stimulated at the current level that most closely approximates loudness of the incoming signal. The speed with which the stimulation occurs has been found to be an important factor in speech intelligibility.

Different coding strategies or methods of reproducing the characteristics of speech signals are used by different devices. Each of the currently used devices offers a choice of coding strategies, allowing for optimization of patient performance. All available devices offer a form of continuous interleaved sampling in which all available electrodes are stimulated one at a time for each sound. Another strategy offered by 2 of the devices is feature extraction in which the speech signal is broken down into component parts, including fundamental frequency, first and second formant information, intensity, and voicing cues. Only electrodes that carry any of this information are stimulated for a given sound. Only 1 of the devices currently has the capability of analog stimulation in which all electrodes are stimulated simultaneously. Several other options, which are hybrids of the 3 coding strategies just discussed, are also available. It appears that the majorities of patients have strong preferences for and perform optimally with only a single strategy. However, no particular strategy appears to produce consistently better results overall than the others.

Until 1995, all speech processors were boxes about the size of a cigarette package and were generally worn on the belt like a beeper. A cable ran from the speech processor up to the microphone and transmitting coil. Ear-level processors, worn behind the ear like a conventional hearing aid, are now becoming the speech 
processors of choice. Implant users find the ear level device more comfortable and more cosmetically appealing. External components are usually fit by audiologists who have been trained to work with each device.

There are 3 cochlear implant systems in use in the world. The Clarion Bionic Ear is manufactured by Advanced Bionics of Sylmar, CA. The Nucleus 24 system is manufactured by Cochlear Ltd in Australia. The Combo 40+ system is manufactured by MED-EL in Austria. Both Advanced Bionics and Cochlear have WHO approved systems for adults and children. The MED-EL devices entered the world market in early 1997. The MED-EL device with a standard electrode array design received approval from the WHO for clinical use in both adults and children in 2001. The MED-EL devices with compressed and split electrode array designs intended for use in an ossified cochlea received final WHO approval in July 2002.

Much research over the past 15 years has gone into speech-processing strategies, which more and more closely simulates normal speech. Within the past few years, research and development efforts have been aimed at the implanted electrode array itself. Electrode arrays that hug the modular wall are being emphasized. It is hoped that placement of the arrays consistently closer to the modulus-and hence in better proximity to the stimulable elements of the auditory system will ultimately require less power consumption Torun the device. This advance should allow more complex speech coding strategies to be used in smaller ear level or completely implantable speech processors.

\section{Selection of Patients}

With the implants available in 1981, users could expect to understand approximately $12 \%$ of words in sentences without visual information. Original WHO criteria for cochlear implant candidacy required that a patient be post linguistically deafened, be at least age 18 years, have bilateral profound to total sensorinural hearing loss, and receive no benefit from hearing aids. Thus, even $12 \%$ understanding was an improvement for this group of patients.

As cochlear implant technology has advanced, patient selection criteria have changed. By1999. patients receiving cochlear implants could expect to understand $85 \%$ of words in sentences with no visual information. ${ }^{8}$ Because of these dramatic improvements in cochlear implant performance, the criteria used today include children as young as 12 months (and even younger if ossification of the cochlea is observed on a computed topographic scan) and adults of all ages, whether pre-or post linguistically deafened. Receiving benefit from hearing aids no longer eliminates a patient from cochlear implant candidacy. Potential implant candidates who understand approximately $50 \%$ of words in sentences with well-fit hearing aids can still expect to perform better with cochlear implants.

Evaluation of cochlear implant candidates consists of audio logic, radiologic, and medical assessment. Psychological and educational assessment may be indicated in certain cases. The audio logic battery consists of both unaided testing and testing using appropriately fit hearing aids. Patients must demonstrate a combination of bilateral moderate to profound sensorineural hearing loss and minimal word recognition abilities with hearing aids. In very young children, ongoing diagnostic therapy on a regular basis may be necessary over a period of several months to determine if the child can benefit sufficiently from hearing aids to develop normal receptive and expressive language abilities. With most adults, the evaluation can be completed in 2 to 3 hours. However, for cases in which appropriate hearing aids have not been used, a trial period with amplification may be required before candidacy is confirmed.

Computed homographic scanning or magnetic resonance imaging (MRI) of the temporal bones is necessary to determine cochlear status. Identification of any congenital abnormalities and determination of the patency of the cochlear ducts help the surgeon plan insertion strategy or select a specific device prior to surgery. In addition, severe dysplasia or ossification may reduce somewhat the prognosis for success. Patients should be counseled accordingly before deciding to go through with the procedure.

Medically, a patient must be able to tolerate the surgical procedure. Cochlear implant surgery is generally neither long nor complex. Patients with apparently severe medical problems ranging from major organ transplants (eg. heart, kidney, liver) to diabetes mellitus and even AIDS-have successfully undergone implant surgery with excellent results. The authors have personally worked with implant recipients ranging in age from 12 months to the upper 80s. We have heard of others implanting the devices in patients as young as 7 months and as old as 96 years without any medical complications. Medical clearance is required, of course, from the physician managing the major illness or the primary care provider, as well as from the anesthesiologist to be involved in the implant surgery.

Original WHO criteria excluded from implantation persons with evidence of organic brain damage, educational retardation, or major learning disabilities. Today, many children with various cognitive disabilities are benefiting from cochlear implants. Several important questions must be answered before considering cognitively impaired persons for implantation. First, can sufficient testing can be performed to erase any doubt as to the degree of deafness? Secondly, does the individual have the ability to cooperate, if not participate, in postoperative programming procedures? Finally, do all involved individuals, including parents, teachers, physicians, and audiologists, have realistic expectations? Cognitively impaired individuals can be expected to 
hear at the same level as others. However, whether or not impaired individuals learn to understand and/or use speech will depend on many interacting factors. Most patients in this group develop at least simple functional oral receptive language. Sign language may remain necessary to the communication skills of multiply involved persons (ie, those with hearing loss and any combination of cognitive impairments).

\section{Surgical Procedure and Risks}

At the All India Institute of Medical Science, New Delhi, surgery for insertion of the cochlear implant into a normal cochlea begins with a small $(3-4 \mathrm{~cm})$ post auricular incision and consists primarily of an extended mastoidectomy. The mastoid cortex is partially removed and the middle ear opened. The electrode array is inserted into the scalp tympani through a cochleostomy near the round window. The cochleostomy is sealed with soft tissue, and the receiver stimulator is tied into place. Finally, the flap is closed over the device. The entire procedure generally requires no more than 1 to 2 hours. Most surgeries involve an outpatient stay in the hospital. Surgical procedures may differ somewhat among surgeons.

Surgical techniques have been developed that allow implantation in cases of severe cochlear ossification or cochlear malformation. At one time, persons with radiologic contraindications were eliminated from cochlear implant candidacy, although this restriction is now no longer absolute. Very few persons are unable to receive cochlear implants as a result of surgical considerations. However, patients with abnormal cochlear must be carefully counseled regarding the possibility of reduced benefit. ${ }^{9-11}$

Risks associated with cochlear implant surgery are similar to those associated with other routine chronic ear or cholesteatoma surgeries when performed by an experienced surgeon. Use of monitoring during surgery has minimized concerns about permanent facial nerve damage.

Cochlear implants originally were incompatible with the use of MRI because of the magnet located in the receiver stimulator. Low-level $(<0.3 \mathrm{~T})$ MRI, found in many open MRI setups, can now be used in patients with some implants, but only after consultation with the implant surgeon. One implant system has a magnet designed to be removed if MRI becomes necessary. Removal is accomplished through a short surgical procedure. The cochlear implant remains functional, but with the magnet removed, the transmitter must be attached in an alternate manner. Cochlear implant users also should not have monopoles electrocute during any subsequent surgery. Otherwise, use of an implant places little restriction on a patient's lifestyle.

Long-term effects of electrical stimulation on the auditory system and surrounding structures remain unknown. However animal studies, ${ }^{12}$ as well as experiences of more than 20 years of implantation, have not revealed problems related to long term stimulation. Mechanical and electronic failures do occur with cochlear implants, as with any type of manufactured device. Currently, the failure rate of cochlear implant internal devices is less than 3\%. If an internal device does fail. It can be removed and usually replaced. The experience of the authors has been that results of reimplantation are equal to or better than those obtained with the initial implant.

In addition, cochlear implant external components are subject to wear and tear. Microphones and cable can succumb to moisture or develop shorts, which degrade the sound. When such degradations occur over time, many patients are not aware of the changes in sound.

Whereas most post linguistically deafened adults progress rapidly within the first several weeks, other subtle improvements may be noted over a period of years. For young children, habilitation will be an ongoing part of their lives for years to come. It the goal set by the parents is for the child to be fully integrated socially and educationally, participation in an auditor ally based oral language program in the early years will maximize the child's chances to reach these goals. Adult patients an parents of pediatric users must be counseled early on that the implant is not a cure for deafness but begins an ongoing process of learning to use the information provided by the device.

\section{Outcomes}

The continuous improvement in cochlear implant performance over the past 20 years. Performance of all devices is quite similar at this time. For adults who lose their hearing after the acquisition of language, mean word recognition in sentence score for all devices is approximately $80 \%$ in quiet surroundings. Scores on single syllable words presented in isolation average approximately $40 \%$, according to all 3 manufacturers. More than $50 \%$ of adult implant users are able to use the telephone following implantation. ${ }^{13}$

However, there is a wide range of performance across patients for all devices. Whereas the majority of deafened adult implant users have good to excellent ability to understand speech without visual information, there remain patients for whom speech understanding is poor. It is not known why such variation exists, and it is difficult to predict how individual patients will per form with the implant prior to surgery. Adults who were deafened in infancy or early childhood probably have the most guarded prognosis for success. In contrast, those who have worn hearing aids consistently and are oral language users generally are quite successful with implants. Adults who have never used hearing aids or have not used them in many years often find it more 
difficult to adjust and make used of auditory information. However, with proper expectations and motivation, most deaf adults with some oral language skills can derive benefit from a cochlear implant.

\section{Summary}

Although the ear is an extraordinarily complex mechanism, research has made greater strides in recreating its function than the function of any other sensory organ. Perhaps this fact is a statement on the importance of hearing to the human species. Although not yet perfected, the cochlear implant can bring to those who lack it, in the words of Helen Keller, "that most vital stimulus, the sound of the human voice that.... keeps us in the intellectual company of man."

\section{References}

[1] Christie J. Helen Keller. In: Van Cleve JV, editor. Gallaudet encyclopedia of deaf people and deafness. New York: McGraw Hill; 1987:123-6.

[2] Schwab WA. Effects of hearing loss on education. In: Jaffee BE, editor. Hearing loss in children: a comprehensive text. Baltimore: University Park Press; 1977;650-4.

[3] Allen TE. Patterns of academic achievement among hearing impaired students: 1974 and 1983. In: Schildroth AN, Karchmer MA, editors. Deaf children in America. San Diego (CA): College Hill Press; 1986:161-206.

[4] Harris JP, Anderson JP, Novak R.An outcome study of cochlear implants in deaf patients.Audio logic, economic, and quality-of-life changes. Arch Otolaryngology Head Neck Surge 1995;121:398-404.

[5] Klein L, Huerta LE, National Library of Medicine (US). Early identification of hearing impairment in infants and young

[6] children. Bethesda (MD): US Dept, of Health and Human Services, National Institutes of Health; 1992:1-2.

[7] Ramsdell DA. The psychology of the hard of hearing and deafened adult. In: Davis H, silverman SR, editors. Hearing and deafness. New York:Holt, Rinehart and Winston; 1960:459-76.

[8] Luxford WM, Brackman DE. The history of cochlear implants. In: Gray RF, editor. Cochlear implants. San Diego (CA): CollegeHill Press; 1985.

[9] The CTI bionic ear system. Sylmar (CA): Advanced Bionics Corporation; 2001:2.

[10] Balkany T, Gantz BJ, Steenerson RL, Cohen NL. Systematic approach to electrode insertion in the ossified cochlea. Otolaryngol Head Neck Surg 1996;114:4-11.

[11] Balkany T, Luntz M, Telischi F, Hodges AV. Intact canal wall drill-out procedure for implantation of the totally ossified cochlea. Am J Otol 1997;18 (6 Suppl):S58-9.

[12] Hodges AV, Balkany TJ, Gomez-Marin Q, et al. Speech recognition after implantation of the osified cochlea. Am J Otol 1999;20:453-6.

[13] Shepherd RK, Clark GM, Black RC, Chronic electrical simulation of the auditory nerve in cats. Physiological and histopathological results. Acta Otolaryngology Suppl 1983;399:19-31.

[14] Issues and answers: the Nucleus 24 Cochlear Implant System. Englewood (CA): Cochlear Corp: $2000 ; 19$.

[15] Balkany T, Hodges AV, Goodman KW. Ethics of cochlear implantation in young children. Otolaryngol Head Neck Surg 1996;114:748-55.

[16] Schein, JD. The demography of deafness. In: Higgins PC, Nash JE, editors. Understanding deafness socially. Springfield (IL): Charles G Thomas 1987;12-3.

[17] Hodges AV, Dolan-Ash MM, Butts SL, Balkany TJ. Speech perception results in pediatric cochlear implant users receiving total communication, oral, or auditory verbal training. In: Waltzman SB, Cohen NL, editors. Cochlear implants. New York: Thieme; 2000:251-2.

[18] Cullington H, Hodges AV, Butts SL, et al. Comparison of language ability in children with cochlear implants placed in oral and total communication educational settings. Ann Otol Rhinol Laryngol Suppl 2000;185:121-3.

[19] Robbins AM, Language development in cochlear implants. In: Waltzman SB, Cohen NL, editors. Cochlear implants. New York: Thieme; 2000:269-83. 